\title{
Cooperativas de viviendas cerradas y diferenciación socio-residencial. El caso de la comuna de Viña del Mar
}

\author{
Carlos Valdebenito Valdebenito* \\ Luis Álvarez Aránguiz* \\ Rodrigo Hidalgo Dattwyler** \\ Carlos Vergara Constela**
}

\section{Resumen}

El artículo presenta la actuación urbana de las cooperativas de vivienda cerrada en la ciudad de Viña del Mar (Chile) durante la última década del siglo XX, las cuales son puestas en diálogo y tensión con los procesos urbanos acaecidos en este territorio. A través de enfoques conceptuales que abordan la ciudad neoliberal y el estudio de la diferenciación socio-residencial del espacio urbano, se busca dar una lectura al lugar que ocupan estas cooperativas. Mediante una metodología cuantitativa descriptiva y multivariada que pretende especificar la construcción de viviendas por parte de las cooperativas y dar cuenta de la distribución espacial de la población respectivamente, se caracteriza el desarrollo de las cooperativas en tanto localización, morfología, superficie construida e inversión, para luego discutir su emplazamiento con algunas de las principales lecturas urbanas sobre la diferenciación socio-residencial. Se concluye con que la huella de las cooperativas de vivienda se difumina y se confunde con el establecimiento de la oferta inmobiliaria privada, donde, si bien los mecanismos de consecución de la vivienda distan en materia de gestión del suelo y asociatividad, ambas formas poseen variadas semejanzas tales como la presencia de espacios públicos privados, el establecimiento de fronteras físicas, la fragmentación de la trama urbana y mecanismos de acceso controlado.

Palabras clave: cooperativas, vivienda, clúster, fragmentación, barrios cerrados.

* Pontificia Universidad Católica de Valparaíso, Valparaíso, Chile.

** Pontificia Universidad Católica de Chile, Santiago, Chile. 


\title{
Gated housing cooperatives and socio-residential differentiation. The case of Viña del Mar community
}

\begin{abstract}
This article presents the urban intervention of gated housing cooperatives in the city of Viña del Mar (Chile) during the last decade of the twentieth century, which are compared and contrasted with urban processes in this territory. By means of conceptual approaches that address the neoliberal city and the study of the socioresidential differentiation of urban space, it is sought to understand the reach of these cooperatives. Through a descriptive and multivariate quantitative methodology that aims to describe the cooperative housing construction and account for the spatial distribution of the respective population, the development of cooperatives is characterized as to location, morphology, constructed area and investment, and then its situation is discussed in the light of some of the main urban approaches to socio-residential differentiation. Findings show that the trajectory of the housing cooperatives is blurred and is confused with the establishment of private real estate offer. That is, despite their different mechanisms for attaining housing regarding land management and associativity, both forms have several similarities such as the presence of private public spaces, the establishment of physical borders, fragmentation of the urban space and controlled access.
\end{abstract}

Keywords: cooperatives, housing, cluster, fragmentation, gated neighborhoods.

\section{Introducción ${ }^{1}$}

iferentes agentes y actorías sociales que se han ocupado de la
construcción formal de la ciudad han disputado la hegemonía de
este campo, buscando direccionar y controlar el diseño normativo y la morfología de ésta (Capel, 2013). Si bien persisten agentes que tuvieron su mayor protagonismo en la fase desarrollista del capitalismo, su capacidad de influencia ha perdido terreno respecto a los agentes que hoy en día se encuentran alineados con los capitales financieros, destinando la ciudad a nichos de acumulación y reproducción de capital (Harvey, 1989; Capel, 2013; Hidalgo; Janoschka, 2014; Hidalgo; Santana; Alvarado, 2016).

${ }^{1}$ Se agradece el apoyo de CONICYT-Chile, a través del Proyecto Fondecyt no 11160747, en la realización de la investigación, algunos de cuyos resultados se comunican en este artículo. 
En las ciudades metropolitanas latinoamericanas podemos apreciar una relación asimétrica de poder entre los múltiples agentes involucrados en las disputas por suelo y vivienda. Actualmente, el mundo privado tiende a controlar la relación con el Estado en materia de producción de espacio urbano. Si bien el Estado ha buscado suplir las necesidades de vivienda con subsidios orientados a la demanda, sus estrategias están principalmente destinadas a operar como creadoras de nichos de reproducción de capital, mediante la promulgación de instrumentos de planificación urbana ad hoc a las necesidades de la reinversión de capital (López-Morales; Gásic; Meza, 2012; Hidalgo; Volker; Ramírez, 2014; Hidalgo et al., 2015). Esto ha implicado la transformación de las metrópolis latinoamericanas, en las cuales se expresan fenómenos socioespaciales como la segregación, la fragmentación y la exclusión social (Hidalgo; Santana; Alvarado, 2016).

No obstante, en este tipo de ciudades existen huellas, a modo de palimpsesto, que dan cuenta del poder que han tenido actorías sociales provenientes de la sociedad civil al momento de diseñar y construir la ciudad. Para estos efectos indagaremos en las acciones que han realizado las cooperativas de vivienda cerrada entre inicios de la década de 1990 y principios de la de 2000 en la comuna de Viña del Mar, Chile. "Viña", como es conocida popularmente, es una comuna costera inserta dentro del área metropolitana de Valparaíso, una de las dos metrópolis que conforman la Macro Región Urbana Central de Chile, espacio donde reside aproximadamente el $80 \%$ de la población del país. Según el último Censo de Población y Vivienda, de 2017, en Viña del Mar habitan 326.759 personas. Es la comuna que más población concentra dentro del área metropolitana de Valparaíso y es la sexta con mayor población dentro del país. En términos administrativos, "Viña" se organiza territorialmente en 12 barrios y 150 unidades vecinales (figura 1 ).

La situación socioeconómica de Viña del Mar tiende a ser contradictoria. Es una comuna donde se localiza población que posee un alto estándar de vida, la cual se ubica principalmente en las zonas costeras. Pero a la vez, es la comuna de Chile que posee mayor cantidad de campamentos (vivienda 
informal), los cuales tienden a ubicarse en laderas y cerros de la ciudad². No obstante, esta expresión socioespacial de la segregación residencial es parte de un proceso que se ha estructurado desde las últimas décadas del siglo pasado hasta la actualidad (Valdebenito, 2011; 2014).

Si bien en otras investigaciones se ha indagado en las estrategias y formas que adopta la producción de hábitat informal (Valdebenito, 2014), en este artículo pondremos énfasis en la acción urbanística de ocho cooperativas cerradas de vivienda emergidas en la última década del siglo veinte, las cuales han sido conformadas mayoritariamente por jóvenes profesionales de la época ${ }^{3}$. Estas cooperativas construyeron ocho proyectos residenciales en Viña del Mar, los cuales se emplazan dentro de tres grandes barrios: Recreo, Plan y Miraflores (figura 1). En total, estos proyectos de vivienda construyeron 1.258 departamentos, representando aproximadamente el $10 \%$ de la producción de vivienda formal de Viña durante la década de los noventa ${ }^{4}$.

\footnotetext{
${ }^{2}$ Según los registros oficiales, el tamaño de la población que reside en asentamientos irregulares en Viña del Mar, agrupados en 64 campamentos, representa aproximadamente el $5 \%$ del total de su población residente, a abril de 2017. Esto representa casi dos puntos porcentuales más de lo catastrado en abril de 2002.

${ }^{3}$ El artículo 1 de la Ley General de Cooperativas, DFL no5 de septiembre de 2003, define a las cooperativas como asociaciones que, de conformidad con el principio de la ayuda mutua, tienen por objeto mejorar las condiciones de vida de sus socios y presentan las siguientes características fundamentales: (i) los socios tienen iguales derechos y obligaciones, un solo voto por persona y su ingreso y retiro es voluntario; (ii) deben distribuir el excedente correspondiente a operaciones con sus socios, a prorrata de aquéllas; (iii) deben observar neutralidad política y religiosa, desarrollar actividades de educación cooperativa y procurar establecer entre ellas relaciones federativas e intercooperativas; y (iv) deben también tender a la inclusión, como asimismo valorar la diversidad y promover la igualdad de derechos entre sus asociadas y asociados. El artículo 74 de la Ley en comento define que son cooperativas de vivienda aquellas que tienen por objeto satisfacer las necesidades habitacionales y comunitarias de sus socios y prestar los servicios inherentes a dicho objetivo. La Ley General de Cooperativas, en su artículo 74, reseña que habrá dos clases de cooperativas de vivienda: las cooperativas cerradas de vivienda, que se organizan para desarrollar un proyecto habitacional, y las cooperativas abiertas de vivienda, que deben ser de objeto único y pueden desarrollar en forma permanente, simultánea o sucesiva diferentes programas habitacionales y tener carácter nacional o bien desarrollar una acción regional. Según las estadísticas oficiales de la Dirección de Obras del Ayuntamiento de Viña del Mar.

${ }^{4}$ Según las estadísticas oficiales de la Dirección de Obras del Ayuntamiento de Viña del Mar.
}

Sociologias, Porto Alegre, ano 22, n. 54, maio-ago 2020, p. 328-356. 
Figura 1 - Localización de las cooperativas cerradas de viviendas en Viña del Mar
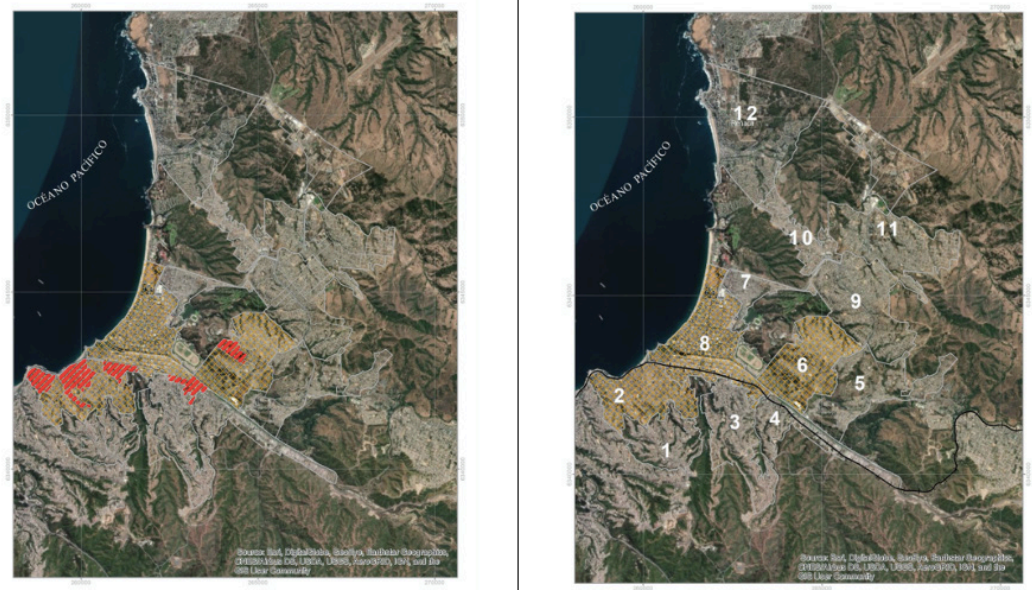

Nota: 1. Nueva Aurora; 2. Recreo; 3. Forestal. 4. Chorrillos; 5. Oriente; 6. Miraflores; 7. Santa Inés; 8. Plan; 9. Achupallas; 10. Gómez Carreño; 11. Reñaca Alto; 12. Reñaca Bajo.

Fuente: Elaboración propia.

En este orden, el objetivo del presente artículo es caracterizar la producción habitacional de estos ocho conjuntos de cooperativas de vivienda cerradas en la comuna de Viña del Mar, dando cuenta de su localización y relación con la estructura socioeconómica de los barrios en que se emplazan, lo que permitirá discutir en torno a la diferenciación social dentro del espacio viñamarino contemporáneo. Para estos efectos, a continuación dará cuenta de los principales enfoques en materia de diferenciación socioeconómica. Luego se expone el diseño metodológico de la investigación. Posteriormente se exponen los principales resultados, los cuales son interpretados a la luz de la diferencia social y económica de los barrios viñamarinos y sus actuales transformaciones urbanas. 


\section{El estudio de la diferenciación socioeconómica del espacio residencial urbano contemporáneo}

Entre las propuestas conceptuales que profundizan en torno a la huella de las intervenciones urbanísticas en la estructura socio-residencial de las ciudades neoliberales destaca la síntesis de Marcuse (1989; 1995; 2011), quien caracteriza la emergencia de la ciudad fracturada en las últimas décadas del siglo XX. Dos son las líneas argumentales postuladas: la primera plantea que las élites tendrían una menor dependencia de los grupos sociales más bajos, pues su vida cotidiana trasciende absolutamente a su lugar de residencia, lo que trae como consecuencia la emergencia de una sociedad crecientemente desconectada, fragmentada y polarizada, socioespacialmente hablando (Arizaga, 2000). La segunda da cuenta del establecimiento de una ciudad dual estructurada bajo los nuevos regímenes de los mercados laborales en áreas metropolitanas, producto de las reestructuraciones económicas del último cuarto del siglo XX. Esta dualidad se basa en la polarización salarial y su expresión espacial de diferencias salariales entre grupos altamente calificados y los que no, bajo una paralela reducción de la clase media.

A la luz de esta argumentación, Marcuse (2011) analiza la estructura social de la ciudad neoliberal y su expresión en el espacio urbano, distinguiendo cinco categorías de habitantes en función de la estructura socio-ocupacional: (i) quienes son propietarios de la riqueza y del poder de decisión; (ii) los profesionales, técnicos y gerentes; (iii) la clase media tradicional, profesional y semiprofesional; (iv) la clase trabajadora tradicional; y (v) los excluidos. Como correlato residencial de esta estructura social y de los procesos de fragmentación, Marcuse (2011) distingue "cinco ciudades dentro de la ciudad", a saber: (i) la ciudad del lujo; (ii) la ciudad gentrificada; (iii) la ciudad suburbana de las clases medias; (iv) la ciudad de los bloques de apartamentos de alquiler con población de clase baja trabajadora; y (v) el gueto. El autor en cuestión particularmente releva el crecimiento de la ciudad gentrificada y de la ciudad abandonada y degradada. Este crecimiento 
se manifestaría en la reconversión en áreas terciarias y en apartamentos ocupados por profesionales, emplazados en áreas relativamente centrales con estados relativos de degradación (Marcuse, 1989).

Específicamente para los casos latinoamericanos, se sostiene que la ciudad ha adoptado una forma urbana con centralidades consolidadas y definidas y expansiones periféricas tipo archipiélago (Bähr; Borsdorf, 2005), la cual sería resultado tanto del asentamiento insular de estructuras y funciones como del aislamiento específico de espacios urbanos a través de la imposición de muros o rejas (Janoschka, 2002). Al igual que Marcuse, se plantea que el principio de estructuración del modelo de ciudad latinoamericana es la fragmentación,

una nueva forma de separación de funciones y elementos socio-espaciales, ya no - como antes - en una dimensión grande (ciudad rica-ciudad pobre; zona habitacional-zona industrial), sino en una dimensión pequeña. Elementos económicos y barrios habitacionales se dispersan y mezclan en espacios pequeños: urbanizaciones de lujo se localizan en barrios muy pobres; centros de comercio se emplazan en todas partes de la ciudad; barrios marginales entran en los sectores de la clase alta. Este desarrollo se hace posible solamente a través de muros y cercos, barreras con que los barrios cerrados se separan y proponen un modo de seguridad que pone como alteridad a las clases populares. Pero hay que mencionar también que el fenómeno de los muros no es privativo de los barrios de la clase alta: los barrios de clase media y baja también se amurallan, y se observa este fenómeno de igual manera en los barrios marginales (Borsdorf, 2003, p. 44).

Siguiendo a Janoschka (2002), podemos apreciar que la ciudad latinoamericana posee estructuras insulares que se han convertido en ejes del desarrollo del espacio urbano. Estas abarcan cuatro dimensiones: islas de riqueza, islas de producción, islas de consumo e islas de precariedad, las cuales se superponen a los ejes radiales de los modelos urbanos previos, donde el elemento estructurante y de unión entre las islas corresponde a las vías de transporte.

Más allá de la discusión específica sobre la morfología de la ciudad latinoamericana, las políticas de liberalización del suelo y mercantilización 
de la vivienda han tenido como efecto la emergencia de procesos de segregación residencial socioeconómica (Ortíz; Schiappacasse, 1998; Vilagrasa, 2000; Rodríguez, 2001; Rodríguez; Arriagada, 2004; Sabatini; Cáceres; Cerda, 2001; Natera; Gómez, 2007; Checa; Arjona, 2007; Sabatini; Brain, 2008), definidos por la actuación de actores y agentes urbanos (Hidalgo; Arenas, 2012; Hidalgo; Zunino, 2011; Valdebenito, 2018). Este último campo de estudios busca explicar y comprender los mecanismos y las lógicas de actuación de actores y agentes urbanos, como medio para comprender de manera más extensa y densa la forma en que la ciudad se construye y los factores más significativos de la organización del espacio urbano (Capel, 2013).

\section{Materiales y métodos}

En términos metodológicos, la investigación presenta dos fases distinguibles. En un primer lugar, la elaboración de una base de datos para dar cuenta de las características de las cooperativas de vivienda en estudio y, en segundo lugar, el análisis de una batería de indicadores que expresan diferencias socioeconómicas en la comuna.

Para efectos de caracterizar las cooperativas en estudio, se solicitaron los registros de los Permisos de Obra Nueva (PON) existentes en la Dirección de Obras Municipales (DOM) de Viña del Mar. Esto permitió identificar variables como la superficie construida en metros cuadrados, año de construcción, número de unidades de viviendas construidas, localización específica e inversión (presupuesto de obra y pago de derechos municipales).

Para el segundo punto se estructuró un diseño para trabajar con un modelo analítico multivariante basado en variables presentes en los Censos de Población y vivienda de 1992 y 2002, las cuales fueron diferenciadas territorialmente según los límites de las unidades vecinales de la comuna 
de Viña del Mar, que se erigieran como unidades de análisis ${ }^{5}$. Con esta base, se proponen 19 indicadores correspondientes a variables laborales y socioeconómicas, educacionales, de maternidad, trabajos de cuidado, dinámica demográfica y hogar y vivienda (tabla 1). A estas variables se les aplicó un análisis factorial con el fin realizar un análisis sintético del número inicial de variables que nos permiten discutir la diferenciación socioeconómica, permitiendo identificar cuáles son las variables específicas que poseen mayor incidencia en su estructuración (Becker, 2012).

El procedimiento del análisis opera mediante correlaciones que reducen la cantidad inicial de variables a una menor cantidad de nuevas variables o factores (Escalante; Caro; Barahona, 2002). El análisis factorial identifica variables subyacentes dentro de un conjunto amplio de variables (Catena; Ramos; Trujillo, 2003) y, de esta manera, nos permitió obtener los componentes principales que definen las diferencias socioeconómicas en la comuna de Viña del Mar.

Las matrices factoriales obtenidas tanto para el año 1992 como para el año 2002 distinguieron cuatro componentes que, en conjunto, explicaban aproximadamente el 80 por ciento de la varianza ${ }^{6}$, lo que se consideró como una solución apropiada a los fines de la presente investigación. Los

\footnotetext{
${ }^{5}$ En su configuración, el municipio de Viña del Mar ha tenido en cuenta la demanda ciudadana y los requisitos genéricos establecidos en el artículo 38 de la Ley 19.418 sobre Juntas de Vecinos y demás Organizaciones Comunitarias, a saber: "[l]as unidades vecinales respectivas serán determinadas por el alcalde, de propia iniciativa o a petición de las juntas de vecinos o de los vecinos interesados, con el acuerdo del Concejo Municipal y oyendo al consejo económico y social comunal, efecto para el cual tendrá en cuenta la continuidad física, la similitud de intereses y otros factores que constituyan el fundamento natural de agrupación de los vecinos. En todo caso, y sin perjuicio de lo que establece el inciso cuarto, al determinar las unidades vecinales, el alcalde procurará que el número de ellas permita la más amplia participación de los vecinos, con el fin de facilitar una fluida relación entre las organizaciones comunitarias y el municipio."

${ }^{6}$ El año 1992, la solución factorial obtenida explicaba el 81,2\% y, el 2002, el 80,6\% de la varianza registrada.
} 
componentes principales correspondieron a nivel socioeconómico ${ }^{7}$, dinámica demográfica $^{\mathbf{8}}$, hogar y vivienda ${ }^{\mathbf{9}}$, y posición en la relación laboral ${ }^{\mathbf{1 0}}$. Dentro de estos, el factor socioeconómico es el que más varianza explicaba en ambos los años.

Con esos nuevos valores, se aplicó un análisis de clúster para distinguir la emergencia de grupos relativamente homogéneos internamente y heterogéneos externamente dentro de las unidades vecinales (unidades de análisis), dando cuenta de la conformación de seis y siete clústeres (1992 y 2002, respectivamente) entre las 138 unidades vecinales de 1992 y las 148 de 2002. Estas soluciones permitieron representar los resultados mediante cartografías que muestran la distribución socioeconómica en Viña del Mar.

${ }^{7}$ Para el año 1992, compuesto por 13 indicadores que explicaban el 37,3\% de la varianza observada, a saber: porcentaje de población de 15 años y más desocupados; porcentaje de población de 15 años y más sin estudios; con estudios básicos y con estudios superiores; porcentaje de población estratificada socioeconómicamente como nivel bajo, nivel medio bajo, nivel medio y nivel alto; porcentaje de mujeres en la fuerza de trabajo ocupada; porcentaje de mujeres menores de 24 años con hijos; porcentaje de población que no nació en la comuna; porcentaje de la población que llego a residir a la comuna en los últimos cinco años; porcentaje de viviendas no permanentes, sin alcantarillado, sin ducha. Para el año 2002, este componente explicó el 28,8\% de la varianza y siendo compuesto por 11 indicadores. En relación con lo registrado el año 1992, en este componente de estratificación socioeconómica no aparecen integrados indicadores relacionados con la vivienda.

${ }^{8}$ Para el año 1992, este componente explicaba el $21,1 \%$ de la varianza siendo compuesto por cinco indicadores, a saber: índice de dependencia de mayores de 64 años, de menores de 15 años; porcentaje de hogares familiares, hogares unipersonales; porcentaje de mujeres que realizan quehaceres en su hogar. El año 2002, este factor explicó el 18,3\% de la varianza observada y está conformado por los mismos cinco indicadores.

${ }^{9}$ Para el año 1992 explicó el 9,7\% de la varianza registrada y está compuesto por três indicadores, a saber: porcentaje de viviendas sin ducha, sin disponibilidad de alcantarillado, sin disponibilidad de agua potable. Este factor, el año 2002, explicó el 18,5\% de la varianza y está compuesto por cuatro indicadores, los mismos de 1992 a los que se le suma el indicador porcentaje de viviendas no permanentes.

${ }^{10}$ Este componente, para el año 1992, explicó el 13,2\% de la varianza observada y está compuesto por quatro indicadores: porcentaje de población de 15 años en la fuerza de trabajo asalariada; porcentaje de población de 15 años y más en la fuerza de trabajo empresaria o patrón; porcentaje de población de 15 años y más desocupada; porcentaje de mujeres en la fuerza de trabajo ocupada. El año 2002, este factor explicó el 14,9\% de la varianza observada y está compuesto también por cuatro indicadores, los que se repiten en relación con lo registrado el año 1992. 
Tabla 1 - Variables seleccionadas para análisis factorial

\begin{tabular}{|l|}
\hline Trabajo y nivel socioeconómico \\
\hline$\%$ de población ocupada de 15 años y más; \\
\hline$\%$ de población desocupada; \\
\hline$\%$ asalariados; \\
\hline$\%$ de empresarios; \\
\hline$\%$ mujeres en la fuerza de trabajo ocupada; \\
\hline$\%$ mujeres que realizan quehaceres en su hogar; \\
\hline$\%$ población estratificada socioeconómicamente como nivel bajo, nivel medio bajo, nivel medio y nivel alto; \\
\hline Educación \\
\hline$\%$ población de 15 años y más sin estudios; \\
\hline$\%$ población con estudios básicos y con estudios superiores; \\
\hline Maternidad \\
\hline$\%$ mujeres menores de 24 años con hijos; \\
\hline Trabajos de cuidado \\
\hline Índice de dependencia de mayores de 64 años, \\
\hline Índice de dependencia menores de 15 años; \\
\hline Dinámica demográfica \\
\hline$\%$ población que no nació en la comuna; \\
\hline$\%$ población que llego a residir a la comuna en los últimos 5 años; \\
\hline Hogar y vivienda \\
\hline$\%$ hogares familiares; $\%$ hogares unipersonales; \\
\hline$\%$ de viviendas no permanentes; \\
\hline$\%$ de viviendas sin conexión a alcantarillado; \\
\hline$\%$ de viviendas sin disponibilidad de agua potable; \\
\hline$\%$ de viviendas sin ducha. \\
\hline
\end{tabular}

Fuente: Elaboración propia en base a Censos de Población y Vivienda 1992 y 2002. 


\section{La diferenciación físico-morfológica del espacio residencial urbano contemporáneo de Viña del Mar}

La forma urbana del área litoral de la metrópolis porteña posee dos componentes estructuradores: las posibilidades de accesibilidad de acuerdo con los planos de pendiente y la acción posterior de creación de infraestructura vial. En esta lógica, la organización física del territorio se ha desarrollado fundamentalmente relacionando la movilidad y el transporte, donde este último implícitamente contiene la variable topográfica. Justamente, en las particulares condiciones de la comuna de Viña del Mar, juega un valor determinante y no puede quedar circunscrito solo al desarrollo de vialidades pues posee más condicionantes, como por ejemplo lo paisajístico.

El plano topográfico caracteriza y determina las condiciones de la ciudad: planos abruptos, estructura acantilada producto de procesos tectónicos sistemáticos y procesos litorales de interfaces frágiles. Esto ha dado como resultado un soporte para el proceso de urbanización accidentado y abisal. Por ejemplo, el promedio de pendientes en la ciudad de Viña del Mar es de $27^{\circ}$, pero en los sectores periurbanos y de proyección urbana este promedio aumenta a $32^{\circ}$. Dicho de otra manera, el desarrollo urbano utiliza fundamentalmente como área de extensión los planos abruptos, no colonizados precedentemente. En Viña del Mar, estos últimos se encuentran entre la segunda y tercera terraza, aproximadamente entre los 100 a 120 msnm y los 180 a 200 msnm (figura 2). En estos tramos se ha concentrado el crecimiento periférico de los últimos 25 años, coincidiendo también con el desarrollo de la infraestructura para ese periodo en las mismas áreas de crecimiento periférico.

De la misma forma como hoy lo hacen las vialidades, desarrollando infraestructura por la periferia y sorteando el plano topográfico, el incipiente conocimiento geomorfológico determinó el emplazamiento del ferrocarril hacia 1857. El trazado privilegió el plano de pendiente de 4-5\% necesario para esa infraestructura, cuya condición era favorable en la cuenca del 
Marga-Marga. Este proceso creó la primera tendencia de suburbanización y expansión urbana en la conurbación Valparaíso-Viña del Mar (Cáceres; Booth; Sabatini, 2002). Los planos moderados del curso inferior de dicha cuenca, en lo sucesivo, posibilitaron conquistar las cimas y laderas de pendiente moderada. No sólo dispuso de condiciones favorables sino que también constituyó el territorio omitido, donde las pendientes pronunciadas, las marginalidades internas, como accidentes geográficos que mantienen las distancias entre las poblaciones, se erigen como causas iniciales para explicar principios físicos de exclusión y segregación (Cáceres; Sabatini, 2007).

Figura 2 - Trama urbana, pendientes y cooperativas cerradas de vivienda en Viña del Mar

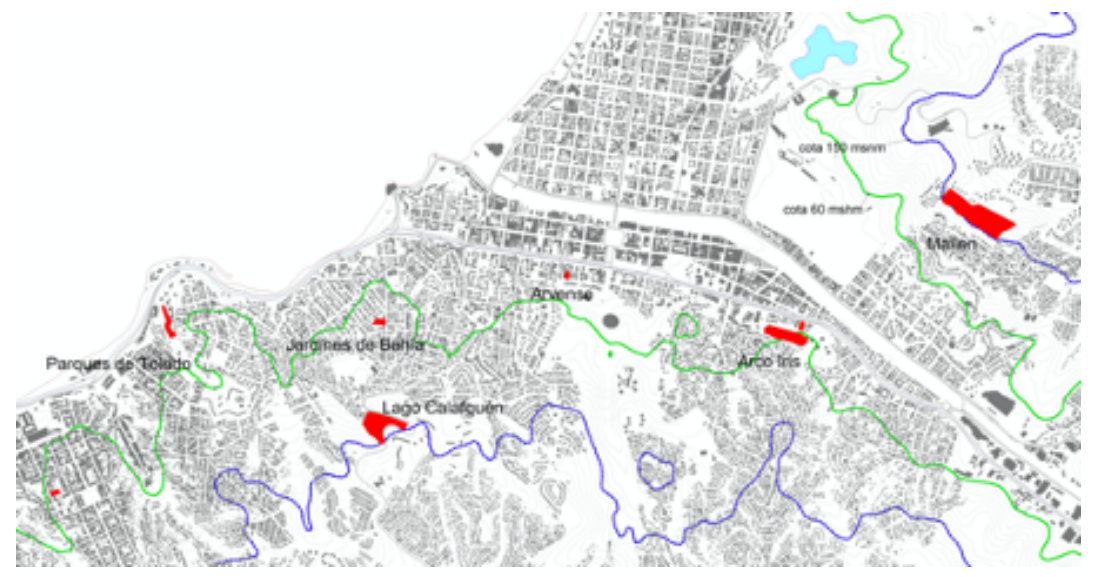

Fuente: Elaboración propia.

La relación de urbanización del plano inclinado siempre ha buscado consolidar los sectores bajos y planos para luego buscar las cimas y reproducir el modelo anterior, donde los intersticios o vacancias entre el plano de borde y la cima superior se consolidan como parques, áreas verdes y/o remanentes de carácter rústico excluidos por pendientes y falta de estructuración. En este marco, proyectos de urbanización de la década de los años sesenta, 
bajo acción estatal, dejaron sustantivos remanentes no urbanizables por la dificultad técnica contemporánea a esos proyectos. Desde la urbanización del Estadio Ferroviario en la Quebrada El Sauce, en 1919, como respuesta pública a la perdida de esos equipamientos en la parte plana del sector Barón, hasta las grandes intervenciones de la Corporación de la Vivienda, entre 1964 y 1976, en las mesetas de Gómez Carreño o la Meseta del Gallo en Miraflores, la intervención pública dejó remanentes no intervenidos (Castagneto, 2010).

Estas urbanizaciones, junto a la remodelación del nuevo acceso a Viña del Mar por la variante Agua Santa, en 1968, fueron proyectos de expansión urbana y creación de hábitat residencial que produjeron subutilización del suelo, debida a la postergación de etapas sucesivas de urbanización por decisiones y acciones políticas posteriores al momento de su desarrollo. Estos territorios cautivos ${ }^{11}$ se han integrado posteriormente a la trama urbana de la ciudad por los siguientes motivos:

i) incremento de la demanda de contexto metropolitano - presión por habitar zonas cercanas al centro, donde la proximidad se ha tornado gravitante producto de la concentración de servicios públicos y privados;

ii) capacidades técnicas y financieras - posibilidad de procesos de reurbanización gracias al avance tecnológico, el cual ha permitido realizar obras imposibles hace 25 años, tales como entibaciones, alzamiento del riesgo y pendiente con obras mayores;

iii) valor paisajístico - aprovechamiento de las potenciales propiedades paisajísticas que podrían producirse a propósito de la urbanización en altura; las vistas de planos de pendientes superiores a los 30 metros sobre el nivel del mar (msnm), durante las últimas décadas, se han constituido en el "valor agregado" de todo proyecto de edificación;

iv) tamaño de la superficie predial - predios cautivos mantenidos como grandes propiedades remanentes de proyectos anteriores. La no subdivisión

"Cuando hablamos de territorios cautivos, hacemos referencia a aquellos que permanecieron en un carácter rústico debido a que la urbanización de determinados conjuntos habitacionales no se completó del todo. 
de estos grandes terrenos, en los cuales no se desarrollaba un uso productivo, permitió traer al presente grandes remanentes, que a través de instancias de compra-venta se han transformado en un recurso inmobiliario;

v) usos de suelo - en general, estos remanentes están declarados como zona de extensión urbana (ZEU) o áreas verdes por riesgos de pendiente; la condición asilvestrada ha permitido mantener una estructura de vegetación nativa xerofita degradada, con proliferación de especies exóticas.

\section{La huella de las cooperativas cerradas de vivienda en del proceso de diferenciación socioeconómica del espacio residencial de Viña del Mar}

La legislación sobre vivienda en Chile se encuentra ajustada a las directrices de un Estado subsidiario. A grandes rasgos, esto quiere decir que el Estado se hace parte cuando no existe oferta privada que cubra el bien o servicio en cuestión, por lo que la provisión de vivienda queda sujeta, predominantemente, a iniciativas privadas (Hidalgo; Santana; Alvarado, 2016). Progresivamente, muchas de estas iniciativas han sido acopladas a sistemas de subsidios a la demanda, en zonas de interés para renovación urbana para la clase media o para la población de menores recursos, sin diferencias respecto a escalas regionales o locales.

Las cooperativas cerradas de vivienda proliferaron como parte de una estrategia privada de consecución de vivienda por parte de grupos profesionales residentes en el área metropolitana de Valparaíso. Estos profesionales se encontraban nucleados en la Federación de Colegios Profesionales Universitarios de Chile, organización que participó en la Asamblea de la Civilidad dentro de la disputa por el derrocamiento de la Dictadura Militar chilena ${ }^{12}$. Este espacio no solo operó como organización

12“Uno de los miembros de la directiva de esta organización multi-gremial era un experto internacional en el campo de las cooperativas que, ya en la década de los setenta, había asesorado la conformación de una cooperativa de vivienda cerrada, orientada a los profesores universitarios de la región de Valparaíso, y la cristalización de su proyecto habitacional. La multi-gremial, conociendo de sus competencias, le encargó la conformación de la primera cooperativa de vivienda cerrada para sus colegiados. El nombre de esta primera cooperativa, Arco Iris, evocaba el pluralismo político de las organizaciones que conformaban la Asamblea de la Civilidad" (Valdebenito, 2018; p.12). 
dispuesta a la lucha política, sino que también como espacio organizacional para la consecución de vivienda por parte de grupos profesionales ${ }^{13}$.

El proceso que va desde la constitución hasta el logro de los objetivos habitacionales de las cooperativas de vivienda cerradas se estructuró en nueve etapas de desarrollo progresivo y acumulativo, en concordancia con los preceptos de la ley que las norma. A saber: 1. Constitución del comité organizador de la cooperativa; 2 . Constitución legal de la cooperativa; 3. Adquisición del terreno en donde emplazar el proyecto habitacional; 4. Concurso y adjudicación del proyecto habitacional; 5 . Obtención del crédito para construir el proyecto habitacional ${ }^{14} ; 6$. Concurso y adjudicación de la construcción del proyecto habitacional; 7 . Construcción del proyecto; 8. Inscripción en el Conservador de Bienes Raíces, tramitación de condiciones de crédito hipotecario a los socios, adjudicación, escrituración y entrega de los departamentos a los socios; y 9. Constitución de la Comunidad de Copropietarios $^{15}$ (Valdebenito, 2018, p. 12).

\section{Entre 1992 y 2002, las ocho cooperativas en estudio construyeron} 1.258 unidades de viviendas en edificios, lo cual representa 176.000 metros cuadrados de superficie, equivalentes a una inversión aproximada de 3.000.000 de Unidades de Fomento (UF) ${ }^{\mathbf{1 6}}$ (tabla 2).

\footnotetext{
${ }^{13} \mathrm{Si}$ bien, originalmente, se pensó en un solo proyecto habitacional (la cooperativa de vivienda cerrada Arco Iris) la cristalización exitosa de ésta, que ya se observa en la adquisición al Estado de Chile de un terreno de su propiedad anexo al Hospital de Viña del Mar a inicios de la década de los años noventa, produjo una expansión de la demanda por este modo cooperativo de resolver el problema de acceso a la vivienda en propiedad, por parte de profesionales y técnicos de estratos medios que no lograron un cupo en la primera iniciativa. Este mismo proceso se desencadenó en los siguientes siete proyectos cooperativos.

${ }^{14}$ La garantía para acceder a este crédito de enlace en el sistema financiero convencional es el terreno adquirido por la cooperativa para construir el proyecto habitacional y, desde luego, la solvencia económica-financiera de sus socios.

${ }^{15}$ Las cooperativas de viviendas cerradas, una vez adjudicados los departamentos y definido el reglamento de copropiedad, se disuelven. Los propietarios conforman una comunidad regida por la Ley 19.537 de Copropiedad Inmobiliaria.

${ }^{16} \mathrm{La}$ unidad de fomento (UF) es una unidad de cuenta usada en Chile, reajustable de acuerdo con la inflación. Fue creada durante el gobierno del presidente Eduardo Frei Montalva mediante el decreto 40 del 20 de enero de 1967 del Ministerio de Hacienda. Su finalidad original era la revalorización de los ahorros de acuerdo con las variaciones de la inflación, permitiendo que el dinero ahorrado en bancos y cajas mantuvieran su poder adquisitivo. Posteriormente, su uso se extendió al sistema crediticio. El monto en comento, a junio de 2018, equivale a 111 millones de euros, aproximadamente.
} 
Tabla 2 - Cooperativas según año de construcción, metros cuadrados construidos, monto de inversión y localización

\begin{tabular}{|l|c|c|c|c|c|c|c|c|}
\hline Cooperativa & $\begin{array}{c}\text { Años } \\
\text { Construcción }\end{array}$ & $\begin{array}{c}\text { Número } \\
\text { Viviendas }\end{array}$ & $\begin{array}{c}\text { Superficie } \\
\text { Construida } \\
(\mathbf{m} \mathbf{)}\end{array}$ & $\begin{array}{c}\text { Monto } \\
\text { Inversión } \\
\text { (UF) }\end{array}$ & $\begin{array}{c}\text { Unidad } \\
\text { Vecinal }\end{array}$ & $\begin{array}{c}\text { Barrio/ } \\
\text { Sector }\end{array}$ & $\begin{array}{c}\text { Clúster } \\
\mathbf{1 9 9 2}\end{array}$ & $\begin{array}{c}\text { Clúster } \\
\mathbf{2 0 0 2}\end{array}$ \\
\hline Arco Iris & $1992-1994$ & 254 & 32.064 & 419.760 & 18 & Plan & 3 & 2 \\
\hline $\begin{array}{l}\text { Parques de } \\
\text { Toledo }\end{array}$ & $1996-1998$ & 122 & 18.400 & 287.508 & 1 & Recreo & 3 & 5 \\
\hline $\begin{array}{l}\text { Lago } \\
\text { Calafquén }\end{array}$ & $1996-1998$ & 250 & 33.802 & 594.490 & 12 & Recreo & 1 & 6 \\
\hline Arvense & $1997-1998$ & 33 & 5.097 & 99.297 & 15 & Plan & 3 & 2 \\
\hline Los Lagos & $1997-1998$ & 61 & 7.596 & 159.082 & 18 & Plan & 3 & 2 \\
\hline $\begin{array}{l}\text { Jardines de } \\
\text { Bahía }\end{array}$ & $1997-1999$ & 59 & 9.300 & 168.754 & 114 & Recreo & 3 & 2 \\
\hline Mallen & $1997-2000$ & 445 & 65.000 & 1.217 .565 & 67 & Miraflores & 2 & 6 \\
\hline Abancay & $2001-2002$ & 34 & 4.700 & 90.000 & 8 & Recreo & 3 & 5 \\
\hline $\begin{array}{l}\text { Viña del } \\
\text { Mar }\end{array}$ & $\mathbf{1 9 9 2 - 2 0 0 2}$ & $\mathbf{1 . 2 5 8}$ & $\mathbf{1 7 5 . 9 5 9}$ & $\mathbf{3 . 0 3 6 . 4 5 6}$ & & & & \\
\hline
\end{tabular}

Fuente: Elaboración propia en base a registros Dirección de Obras Municipales de Viña del Mar.

Tal como podemos apreciar en la tabla 3, en el periodo intercensal 1992-2002, el parque de viviendas permanentes aumentó notablemente en Viña del Mar. Resulta llamativo que la actividad constructiva se concentrase predominantemente en cuatro barrios, dentro de los cuales se ubican dos de los barrios en que se han implementado los proyectos de las cooperativas de vivienda cerrada (Recreo y Plan). Más aún, lo interesante de la estadística presentada es que el crecimiento del parque habitacional no necesariamente posee un correlato poblacional, cuestión que permite interpretar que el crecimiento de proyectos en determinados barrios posee más una lógica de producción de segunda residencia que de suplir u orientar la oferta hacia la demanda habitacional existente en la comuna de Viña del Mar y el área metropolitana (tabla 4). Los datos permiten apreciar un crecimiento poblacional dispar según zonas de la ciudad, aunque en términos generales da cuenta de una ralentización de la dinámica demográfica. 
Tabla 3 - Barrios según tamaño y crecimiento del parque de viviendas particulares

\begin{tabular}{|l|c|c|c|c|c|c|}
\hline \multirow{2}{*}{\begin{tabular}{c}
\multirow{2}{*}{$\begin{array}{c}\text { Área de } \\
\text { Estimación }\end{array}$} \\
\cline { 2 - 7 }
\end{tabular}} & $\begin{array}{c}|c| \\
\text { Tipo } \\
\text { Departamento }\end{array}$ & $\begin{array}{c}\text { Tipo } \\
\text { Casa }\end{array}$ & $\begin{array}{c}\text { Tipo } \\
\text { Departamento }\end{array}$ & $\begin{array}{c}\text { Tipo } \\
\text { Casa }\end{array}$ & Depto. & \multicolumn{2}{c|}{$\begin{array}{c}\text { Tasa Crecimiento } \\
1992-2002\end{array}$} \\
\hline Nueva Aurora & 349 & 3.713 & 1.051 & 4.430 & 11,02 & 1,77 \\
\hline Recreo & $\mathbf{1 . 8 8 1}$ & $\mathbf{5 . 9 9 1}$ & $\mathbf{3 . 4 9 9}$ & $\mathbf{5 . 9 2 1}$ & $\mathbf{6 , 2 1}$ & $\mathbf{- 0 , 1 2}$ \\
\hline Forestal & 731 & 6.373 & 1.423 & 7.357 & 6,66 & 1,44 \\
\hline Chorrillos & 52 & 2.086 & 202 & 2.205 & 13,57 & 0,55 \\
\hline Oriente & 1.378 & 6.467 & 1.784 & 6.522 & 2,58 & 0,08 \\
\hline Miraflores & $\mathbf{2 . 7 3 6}$ & $\mathbf{5 . 0 8 9}$ & $\mathbf{3 . 1 8 1}$ & $\mathbf{5 . 2 2 3}$ & $\mathbf{1 , 5 1}$ & $\mathbf{0 , 2 6}$ \\
\hline Santa Ines & 946 & 2.621 & 1.265 & 2.647 & 2,91 & 0,1 \\
\hline Plan & $\mathbf{1 1 . 3 1 0}$ & $\mathbf{5 . 7 0 0}$ & $\mathbf{1 3 . 5 5 7}$ & $\mathbf{4 . 9 2 4}$ & $\mathbf{1 , 8 1}$ & $\mathbf{- 1 , 4 6}$ \\
\hline Achupallas & 50 & 6.269 & 962 & 8.723 & 29,57 & 3,3 \\
\hline Gómez Carreño & 1.925 & 3.366 & 2.444 & 3.416 & 2,39 & 0,15 \\
\hline Reñaca Alto & 110 & 2.918 & 1.764 & 5.456 & 27,75 & 6,26 \\
\hline Reñaca Bajo & 2.332 & 2.492 & 5.305 & 2.946 & 8,22 & 1,67 \\
\hline Viña del Mar & $\mathbf{2 3 . 8 0 0}$ & $\mathbf{5 3 . 0 8 5}$ & $\mathbf{3 6 . 4 3 7}$ & $\mathbf{5 9 . 7 7 0}$ & $\mathbf{4 , 2 6}$ & $\mathbf{1 , 1 9}$ \\
\hline
\end{tabular}

Fuente: Elaboración propia a partir de la información censal procesada a través de REDATAM R+.

Podemos apreciar que, en Viña del Mar, el modo predominante de urbanización formal corresponde al incremento de la superficie habitable a través de la densificación de las áreas centrales o pericentrales, con especial énfasis en el borde costero y en los lugares donde la edificación en altura permite capturar atributos paisajísticos (Hidalgo; Arenas, 2012). Las cooperativas de vivienda en estudio tienden a ubicarse en zonas centrales, con atributos de proximidad a equipamientos y comercios y, en algunos casos, con aprovechamiento de vistas al mar.

Si bien las cooperativas cerradas se asemejan a las inmobiliarias en tanto adoptan la estrategia de densificación en áreas centrales y pericentrales, se distinguen en dos asuntos: el marco procedimental de obtención de la vivienda (auto organización de la demanda) y su localización específica. 
Tal como muestra la literatura reciente, la forma de densificación dominante desplegada por los agentes inmobiliarios privados da cuenta de la densificación de predios - soportada por recursos jurídicos, como la "fusión predial", contenidos en la legislación urbana vigente - con efectos de modificación de la morfología urbana, acción orientada a la captura de altos volúmenes de renta de suelo (Brenner; Marcuse; Mayer, 2011; López-Morales; Gásic; Meza, 2012; Hidalgo et al., 2015). En este sentido, la gran diferencia que posee la construcción de viviendas por parte de cooperativas es la densificación habitacional en los predios remanentes que dejó la acción estatal en la creación de conjuntos habitacionales planificados durante décadas previas a la dictadura militar.

Tabla 4 - Barrios según tamaño y crecimiento poblacional / 1992-2002

\begin{tabular}{|l|c|c|c|c|c|c|}
\hline $\begin{array}{c}\text { Área de } \\
\text { Estimación }\end{array}$ & $\begin{array}{c}\text { Población } \\
\text { residente }\end{array}$ & $\begin{array}{c}\text { Población } \\
\text { residente }\end{array}$ & $\begin{array}{c}\text { Población } \\
\text { residente }\end{array}$ & ICE & ICE & $\begin{array}{c}\text { Tasa de } \\
\text { Crecimiento } \\
\text { Anual }\end{array}$ \\
\cline { 2 - 7 } & 1982 & 1992 & 2002 & $1982-1992$ & $1992-2002$ & $1992-2002$ \\
\hline Nueva Aurora & 18.021 & 17.915 & 17.499 & $-0,3$ & $-1,2$ & $-0,23$ \\
\hline Recreo & $\mathbf{2 9 . 0 9 3}$ & $\mathbf{2 9 . 0 0 9}$ & $\mathbf{2 7 . 5 1 4}$ & $\mathbf{- 0 , 1}$ & $\mathbf{- 2 , 6}$ & $\mathbf{- 0 , 5 3}$ \\
\hline Forestal & 29.481 & 33.533 & 30.405 & 6,4 & $-4,9$ & $-0,98$ \\
\hline Chorrillos & 7.744 & 9.110 & 7.654 & 8,1 & $-8,7$ & $-1,74$ \\
\hline Oriente & 19.560 & 28.781 & 26.316 & 19,1 & $-4,5$ & $-0,9$ \\
\hline Miraflores & $\mathbf{2 8 . 4 3 7}$ & $\mathbf{3 1 . 2 8 2}$ & $\mathbf{2 7 . 0 9 2}$ & $\mathbf{4 , 8}$ & $-\mathbf{- 7 , 2}$ & $\mathbf{- 1 , 4 4}$ \\
\hline Santa Ines & 13.986 & 14.997 & 13.539 & 3,5 & $-5,1$ & $-\mathbf{1 , 0 2}$ \\
\hline Plan & $\mathbf{4 2 . 4 9 8}$ & $\mathbf{4 1 . 6 0 1}$ & $\mathbf{4 1 . 5 2 1}$ & $\mathbf{- 1 , 1}$ & $\mathbf{- 0 , 1}$ & $\mathbf{- 0 , 0 2}$ \\
\hline Achupallas & 22.920 & 29.518 & 35.056 & 12,6 & 8,6 & 1,72 \\
\hline $\begin{array}{l}\text { Gómez } \\
\text { Carreño }\end{array}$ & 19.920 & 20.919 & 19.504 & 2,4 & $-3,5$ & $-0,7$ \\
\hline Reñaca Alto & 12.112 & 14.584 & 24.990 & 9,3 & 26,3 & 5,39 \\
\hline Reñaca Bajo & 5.205 & 12.562 & 14.954 & 41,4 & 8,7 & $\mathbf{1 , 7 4}$ \\
\hline Viña del Mar & $\mathbf{2 4 9 . 5 4 8}$ & $\mathbf{2 8 5 . 4 5 4}$ & $\mathbf{2 8 6 . 9 3 1}$ & $\mathbf{6 , 7}$ & $\mathbf{0 , 3}$ & $\mathbf{0 , 0 5}$ \\
\hline
\end{tabular}

Fuente: Elaboración propia a partir de la información censal procesada a través de REDATAM R+. 
La estrategia de densificación, desplegada por los agentes inmobiliarios, ha tendido a concentrarse en la zona del borde costero y en barrios como Plan y Recreo. Eso ha modificado el patrón de asentamiento existente y ha provocado efectos negativos como la saturación de ejes viales, la consolidación de patrones de segregación residencial socioeconómica, degradación ambiental y pérdida de valores paisajísticos (Valdebenito, 2011).

El crecimiento regular, ya sea en expansión urbana o densificación en zonas centrales, ha tendido a fragmentar el espacio urbano. Lo sucedido en el centro histórico, dentro del polígono en que convergen las unidades vecinales que conforman la Población Vergara, sirve para graficar el proceso de expansión del centro de la ciudad por todo el Plan, mediante la instalación de actividades de comercio, servicios y proyectos inmobiliarios orientados tanto a hogares permanentes como a la renta turística, hogares para estudiantes universitarios y segunda residencia; orientados a sectores socioeconómicos medio-alto y alto, dirigidos predominantemente a profesionales jóvenes, estudiantes y/o uso turístico. Si bien las transformaciones urbanísticas, socioeconómicas y demográficas han modificado la estructura socioresidencial de la ciudad, estas han sido parciales, lo que se evidencia en los indicadores socioeconómicos, demográficos y habitacionales de sus unidades vecinales (figura 3).

La expresión socioeconómica y demográfica de las unidades vecinales donde se localizan las cooperativas de viviendas cerradas se ve expresada en la continuidad o ruptura, con relación a la configuración de 1992, de áreas socialmente homogéneas en el año 2002 (tabla 5). Los cambios más significativos en las estructuras territoriales se observan en las unidades vecinales que alojaron a las cooperativas Parques de Toledo, Lago Calafquén y Abancay.

En el caso de las unidades vecinales 1 y 8 de Recreo, transitan desde un clúster donde predominan los grupos socioeconómicos medio y mediobajo (clúster 3 de 1992 y 2 de 2002) a un clúster en donde predominan los hogares de estrato medio-alto (clúster 6 de 1992 y 5 de 2002). En el caso de la unidad vecinal 12 de Recreo es más llamativo, en tanto que el 
nivel socioeconómico del barrio cambia completamente, pues la zona en cuestión transita desde un clúster en donde predominan los hogares de estrato bajo (clúster 1 de 1992 y 2002) a otro en donde predominan los hogares de estrato alto (clúster 6 de 2002). Sin embargo, podemos encontrar una explicación de este abrupto cambio, ya que esta zona de Agua Santa se encontraba escasamente poblada en 1992, siendo el mismo proyecto Lago Calafquen una edificación pionera que detonó el interés de agentes inmobiliarios por comprar terrenos y edificar en torno a esta vía que une el plan con los cerros de Viña del Mar (Nueva Aurora, Villa Monte, Recreo Alto) y de Valparaíso (Esperanza, Los Placeres, Rodelillo).

Figura 3 - Unidad vecinal según clúster socioeconómico y demográfico / 1992-2002

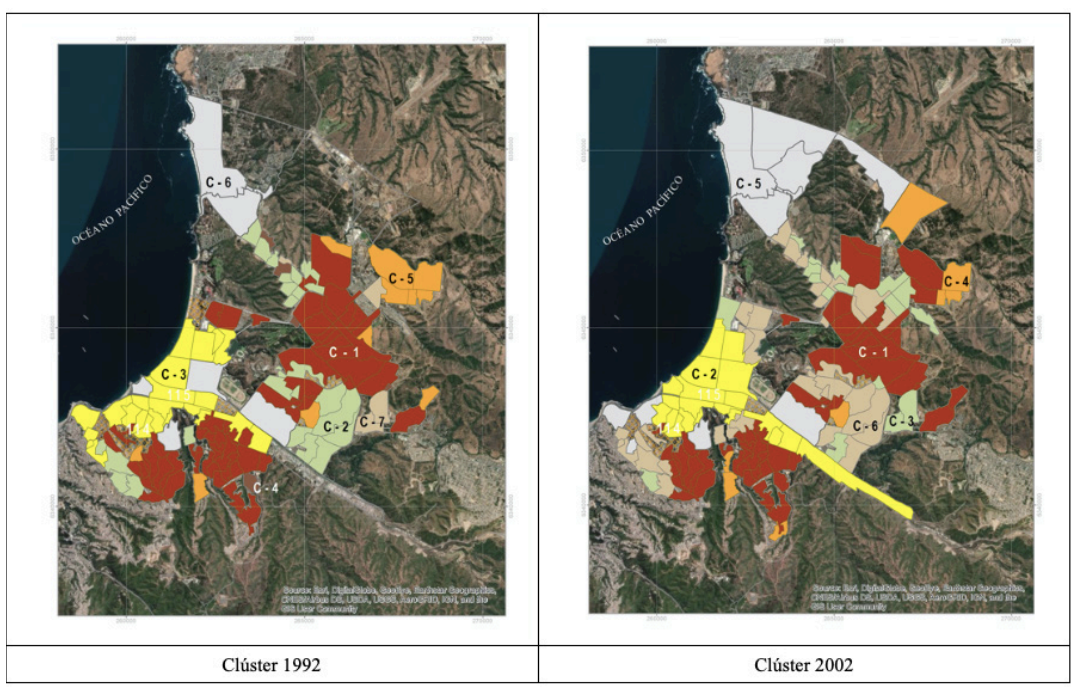

Fuente: Elaboración propia. Información censal procesada a través de REDATAM R*, SPSS y ArcGIS 9.0. 
Tabla 5 - Cooperativas según unidad vecinal, barrio y clúster /1992-2002

\begin{tabular}{|l|c|c|c|c|}
\hline \multicolumn{1}{|c|}{ Cooperativa } & Unidad Vecinal & Barrio / Sector & Clúster 1992 & Clúster 2002 \\
\hline Arco Iris & 18 & Plan & 3 & 2 \\
\hline Parques de Toledo & 1 & Recreo & 3 & 6 \\
\hline Lago Calafquén & 12 & Recreo & 3 & 2 \\
\hline Arvense & 15 & Plan & 3 & 2 \\
\hline Los Lagos & 18 & Plan & 3 & 6 \\
\hline Jardines de Bahía & 114 & Recreo & 2 & 5 \\
\hline Mallen & 67 & Miraflores & 3 & 2 \\
\hline Abancay & 8 & Recreo & & 2 \\
\hline
\end{tabular}

Fuente: Elaboración propia.

En el caso de las cooperativas Arco Iris, Arvense y Los Lagos, ubicadas en el Plan, continúan perteneciendo a un clúster de unidades vecinales caracterizado socioeconómicamente, tanto en 1992 como en 2002, por registrar la presencia de hogares de estrato medio-bajo (clúster 3 de 1992 y 2 de 2002). La unidad vecinal 67 de Miraflores, donde se emplaza la cooperativa Mallén, varía significativamente su condición socioeconómica, siendo parte de un clúster que se caracteriza por contener una más alta prevalencia de población estratificada socioeconómicamente como mediaalta en 2002.

\section{Discusión y cierre}

En términos espaciales, las densificaciones del sistema cooperativo cerrado han sacado provecho de los remanentes de proyectos incompletos heredados de la acción deliberada modernizadora, fomentada por el Estado y llevada a cabo por los recursos generados por las cajas previsionales y las acciones de creación de infraestructura públicas durante las décadas previas a la dictadura militar. Los proyectos asimilan estos remanentes con modelos y formas recientes en patrones de construcción. La densificación, en cierto 
modo, permite completar la trama urbana cortada abruptamente por el plano topográfico. De todas maneras, las cooperativas de vivienda cerrada también erigieron barrios cerrados, con acceso controlado y fragmentación de la trama urbana, en tanto no generaron equipamiento público o no han favorecido la integración formal de lo que originalmente se entendía como el parque de la urbanización.

Las cooperativas de viviendas cerrada, si bien no se diferencian significativamente de otros agentes inmobiliarios en el artefacto urbanístico adoptado en la cristalización de las prácticas de densificación residencial (el condominio cerrado), sí lo han hecho en su localización, en el tipo de suelo ocupado y, sobre todo, en la participación de sus habitantes dentro de las decisiones más estructurales de los proyectos habitacionales.

Si a ello sumamos su concentración en solo siete de las aproximadamente 150 unidades vecinales en que se estructura espacialmente la ciudad, podemos afirmar fundadamente que la intervención de las cooperativas de vivienda cerrada entre 1992 y 2002, que representó alrededor del 10\% de la edificación habitacional formal, ha dejado una huella en la geografía social de la ciudad y, en especial, en las unidades vecinales y barrios en donde se localizaron, logrando al menos contener el éxodo de su población de estratos socioeconómicos medios, erigiéndose en una alternativa para hacer posible la residencia en la ciudad de estos grupos.

Esta alternativa de gestión inmobiliaria, cristalizada en la construcción de ocho grandes proyectos inmobiliarios, emplazados en suelos urbanos vacantes en los barrios Recreo, Plan y Miraflores, que benefició a 1.258 hogares y aproximadamente cinco mil personas, perdió fuerza y vigor en la primera década del siglo XXI, si la comparamos con el accionar inmobiliario, que ha dominado la oferta residencial y ha financiarizado el acceso a la vivienda mediante mecanismos crediticios (Hidalgo; Janoschka, 2014; Gásic, 2018). La huella de las cooperativas de vivienda se difumina y se confunde con el establecimiento de la oferta inmobiliaria privada. Si bien los mecanismos de consecución de la vivienda distan en materia de gestión del suelo y asociatividad, poseen variadas semejanzas: espacios públicos 
privados, establecimiento de fronteras físicas, fragmentación de la trama urbana, mecanismos de acceso controlado y tecnologías de vigilancia (Carman; Vieira; Segura, 2013).

Por otro lado, las asociaciones de profesionales que dieron origen a estas cooperativas de vivienda cerrada poseen un marcado carácter de clase media y media alta. Si bien no se ha realizado una aproximación cualitativa mediante relatos sobre las trayectorias biográficas de quienes participaron en estos proyectos, las generaciones de profesionales que se formaron durante la década de los ochenta han sido excepcionales en tanto han podido experimentar la movilidad social ascendente debido a su condición universitaria (Mac-Clure, 2012). Dicho de otra manera, los grupos sociales que posibilitaron estas cooperativas son bastante homogéneos en su condición socioeconómica, debido a su trayectoria profesional. A priori, esto permite interpretar que no existía una disposición a la mezcla y la mixtura con otros grupos sociales que también requerían solucionar problemas de vivienda, como lo sucedido en Copenhague, Dinamarca (Boterman, 2012). En este sentido, estos grupos de profesionales tuvieron la capacidad de poder decidir dónde deseaban desarrollar sus vidas y estructurar su hábitat residencial, mediante la creación de fronteras físicas que posibilitaban un disfrute interno y exclusivo de espacios públicos u otra infraestructura (Aguilar; Mateos, 2011).

En la actualidad, los barrios donde se ubican las cooperativas de vivienda cerrada en estudio han tendido a elitizarse y disminuir su heterogeneidad social. Por ejemplo, en las unidades vecinales ubicadas en Recreo, vemos que ha existido cierto repoblamiento basado en el arribo de población vinculada a nuevos profesionales y estudiantes que migran hacia la quinta región para desarrollar estudios. Esta es una de las zonas de Viña del Mar donde existe mayor dinamismo en el mercado de la vivienda y en la oferta privada. A la par, se identifica que este barrio posee atributos como el paisaje y la proximidad, los cuales se articulan como plusvalías urbanas y valores agregados (Miralles-Guasch; Sardà, 2014). Justamente, la ubicación de Recreo en medio de la conurbación costera permite tener accesibilidad 
hacia carreteras interurbanas que permiten conectarse con la capital o con otras comunas del área metropolitana, además de poseer notable cercanía tanto hacia el centro de Valparaíso como el de Viña del Mar. Incluso, el proyecto Lago Calafquén es pionero en la urbanización del remanente de suelo ubicado entre Recreo y Nueva Aurora, hecho que permitió la continuidad de la urbanización mediante oferta inmobiliaria privada en torno al eje vial Agua Santa.

Por otra parte, los límites de las unidades vecinales (unidades de análisis) no necesariamente permiten apreciar las fronteras simbólicas de los cerros, como para definir patrones espaciales de segregación residencial socioeconómica. Por ejemplo, la zona donde se ubica la Cooperativa Mallén (Miraflores), de alguna forma tiende a establecer la marca entre la zona "pudiente" y la zona "popular" de este cerro viñamarino. Esto nos obliga a problematizar las unidades de análisis cuando se identifican estos fenómenos, ya que las unidades vecinales y las zonas censales permiten desagregar el espacio comunal pero no necesariamente existe homología con el establecimiento de límites que marcan apropiaciones barriales, fronteras y/o cambios en los imaginarios urbanos dominantes. De hecho, las fronteras simbólicas tienden a persistir sobre otro tipo de fronteras, cuestión que obliga a realizar aproximaciones cualitativas al fenómeno de la segregación residencial, con el fin de complejizar la mera identificación de la distribución espacial de grupos sociales, bienes y servicios (Sabatini; Cáceres; Cerda, 2001; Rodríguez, 2001), ya que estos procesos están basados en formas de clasificación e imaginarios de distinción social (Prévot-Schapira, 2001).

En definitiva, la acción de las cooperativas de vivienda cerrada se confunde con el accionar de la oferta inmobiliaria privada. Su localización en zonas de renovación urbana y en lugares donde se urbanizó para completar la trama de la ciudad, en conjunto con el diseño de los nuevos hábitat residenciales, nos permite establecer que la decisión de comprar suelo en barrios con buenos atributos urbanos (proximidad y paisaje), en conjunto con la disposición a cercar y, con ello, establecer mecanismos de seguridad tales como vigilancia y acceso controlado, han sido pioneros 
en la fragmentación urbana de Viña del Mar, puesto que estos proyectos habitacionales han creado espacios herméticos, con espacios públicos privados compuestos por habitantes con altos niveles de calificación, que se establecen en barrios que han tendido a homogeneizarse en dichos parámetros, afectando la apropiación y uso del espacio urbano por diferentes grupos sociales.

Carlos Valdebenito-Valdebenito es docente e Investigador en Pontifica Universidad Católica de Valparaíso.

ఏcarlos.valdebenito@pucv.cl

Luis Álvarez Aránguiz es docente e Investigador en Pontifica Universidad Católica de Valparaíso.

$\ggg$ luis.alvarez@pucv.cl

Rodrigo Hidalgo Dattwyler es Docente e Investigador en Pontifica Universidad Católica de Chile.

$\equiv$ rhidalgd@uc.cl

Carlos Vergara Constela es Doctorando en Geografía en la Pontifica Universidad Católica de Chile.

$\Xi$ cdvc87@gmail.com

\section{Referencias}

1. AGUILAR, Adrián; MATEOS, Pablo. Diferenciación sociodemográfica del espacio urbano de la Ciudad de México. Revista EURE, v. 37, n. 110, p. 5-30, 2011. http://dx.doi.org/10.4067/S0250-71612011000100001

2. ARIZAGA, María. Murallas y barrios cerrados. La morfología espacial del ajuste en Buenos Aires. Nueva Sociedad, n. 166, p. 22-32, 2000.

3. BÄHR, Jurgen; BORSDORF, Axel. La ciudad latinoamericana. La construcción de un modelo. Vigencia y perspectivas. Ur[b]es, v. 2, n. 2, p. 207-221, 2005.

4. BECKER, Howard. Outsiders. Hacia una sociología de la desviación. Buenos Aires: Siglo XXI Editores, 2012.

5. BORSDORF, Axel. Cómo modelar el desarrollo y la dinámica de la ciudad latinoamericana. Revista EURE, v. 29, n. 86, p. 37-49, 2003.

6. BRENNER, Neil; MARCUSE, Peter; MAYER, Margit. Cities for people, not for profit - critical urban theory and the right to the city. Londres: Routledge, 2011.

7. BOTERMAN, Willem. Deconstructing coincidence: how middle-class households use various forms of capital to find a home. Housing, Theory and 
Society, v. 29, n. 3, p. 321-338, 2012. https://doi.org/10.1080/14036096.2011. 638315

8. CÁCERES, Gonzalo; BOOTH, Rodrigo; SABATINI. Francisco. Suburbanización y suburbio en Chile: una mirada al Gran Valparaíso decimonónico (1820-1870). Archivum, n. 4, p. 151-164, 2002.

9. CÁCERES, Gonzalo; SABATINI, Francisco. Suburbanización y segregación urbana en el Chile decimonónico: hipótesis sobre la formación histórica del Gran Valparaíso. In: VALENZUELA, Jaime. Historias urbanas. Homenaje a Armando de Ramón. Santiago: Ediciones Universidad Católica de Chile, 2007. p. 93-121.

10. CAPEL, Horacio. La morfología de las ciudades. III - Agentes urbanos y mercado inmobiliario. Barcelona: Ediciones del Serbal, 2013.

11. CARMAN, María; VIEIRA, Neiva; SEGURA, Ramiro. Antropología, segregación y diferencia. In: CARMAN, María; VIEIRA, Neiva; SEGURA, Ramiro. Segregación y diferencia en la ciudad. Quito: CLACSO - FLACSO, 2013. p.11-36.

12. CASTAGNETO, Pietro. Una historia de Viña del Mar: la hija de los rieles. RIL Editores: Santiago, 2010.

13. CATENA Andrés; RAMOS Manuiel; TRUJILLO, Humberto. Análisis multivariado. Un manual para investigadores. Madrid: Editorial Biblioteca Nueva, 2003.

14. CHECA, Juan; ARJONA, Ángeles. Factores explicativos de la segregación residencial de los inmigrantes en Almería. Revista Internacional de Sociología, v. 65, n. 48, p. 173-200, 2007.

15. ESCALANTE, Eduardo; CARO, Alberto, BARAHONA, Luis. Análisis y tratamiento de datos en SPSS. Valparaíso: Ediciones Universidad de Playa Ancha 2002.

16. GÁSIC, Ivo. Inversiones e intermediaciones financieras en el mercado de suelo urbano. Principales hallazgos a partir de transacciones de terrenos en Santiago de Chile, 2010-2015. Revista EURE, v. 44, n. 133, p. 29-50, 2018. http://dx.doi. org/10.4067/s0250-71612018000300029

17. HARVEY, David. From managerialism to entrepreneurialism: the transformation in urban governance in late capitalism. Geografiska Annaler, Series B, Human Geography, v. 71, n. 1, p. 3-17, 1989.

18. HIDALGO, Rodrigo; ARENAS, Federico. Negocios inmobiliarios en el frente litoral del Área Metropolitana de Valparaíso (AMV): entre la (des)protección del medio natural y la conservación del patrimonio cultural de la UNESCO. In: XII Coloquio Internacional de Geocrítica, 7 a 11 de mayo de 2012, Bogotá. Anales... Bogotá: Universidad Nacional de Colombia, 2012.

19. HIDALGO, Rodrigo; JANOSCHKA, Michael. La ciudad neoliberal. Gentrificación y exclusión en Santiago de Chile, Buenos Aires, Ciudad de México y Madrid. Santiago de Chile: Pontificia Universidad Católica de Chile, 2014. 
20. HIDALGO, Rodrigo; ZUNINO, Hugo. La urbanización de las áreas periféricas en Santiago y Valparaíso: el papel de las relaciones de poder en el dibujo de la geografía socioresidencial. Revista EURE, v. 37, n. 111, p. 79-105, 2011. http:// dx.doi.org/10.4067/S0250-71612011000200004

21. HIDALGO, Rodrigo; SANTANA, Daniel; ALVARADO, Voltaire. Mitos, ideologías y utopías neoliberales en la producción del espacio: hacia una agenda de investigación alternativa". In: HIDALGO, Rodrigo et al. En las costas del neoliberalismo. Naturaleza, urbanización y producción inmobiliaria: experiencias en Chile y Argentina. Santiago de Chile: Serie Geo Libros, 2016. p. 24-66.

22. HIDALGO, Rodrigo; VOLKER, Pascal; RAMÍREZ, Natalia. La ciudad inmobiliaria: mecanismos institucionales, relaciones de poder y mercantilización del medio natural. El caso del área metropolitana de Valparaíso. Scripta Nova Revista Electrónica de Geografía y Ciencias Sociales, v. 43, n. 493, art. 34, 2014.

23. HIDALGO, Rodrigo; ALVARADO, Voltaire; VOLKER, Pascal; ARENAS, Federico; SALAZAR, Alejandro. Ordenamiento costero metropolitano en Chile: de la expectativa de regulación a la planificación cooptada (1965-2014). Cuadernos de Vivienda y Urbanismo, v. 8, n. 16, p. 206-225, 2015. https://doi. org/10.11144/Javeriana.cvu8-16.ocmc

24. JANOSCHKA, Michael. El nuevo modelo de la ciudad latinoamericana: fragmentación y privatización. Revista EURE, v. 28, n. 85, p. 11-20, 2002. http:// dx.doi.org/10.4067/S0250-71612002008500002

25. LÓPEZ-MORALES, Ernesto; GÁSIC, Ivo; MEZA, Daniel. Urbanismo proempresarial en Chile: políticas y planificación en la producción residencial en altura en el pericentro del Gran Santiago. Revista INVI, v. 27, n.76, p.75-114, 2012. http://dx.doi.org/10.4067/S0718-83582012000300003

26. MAC-CLURE, Óscar. Las nuevas clases medias en Chile: un análisis de cohortes. Revista CEPAL, n. 108, p. 169-182, 2012.

27. MARCUSE, Peter. Dual city: a muddy metaphor for a quartered city. International Journal of Urban and Regional Research, v. 13, n. 4, p. 697-708, 1989. https://doi.org/10.1111/j.1468-2427.1989.tb00142.x

28. MARCUSE, Peter. Not chaos but walls: potsmodernism and the partitioned city. In: WATSON, Sophie; GIBSON, Katherine. Postmodern cities and spaces. Oxford: Blackwell, 1995. p. 243-253.

29. MARCUSE, Peter. Enclaves yes, ghettoes no. Segregation and the State. In: International Seminar on the Segregation of the City, 26 a 28 de julio, 2001, Boston. Anales... Boston: Lincoln Institute of Land Policy, 2011.

30. MIRALLES-GUASCH, Carme; SARDÀ, Oriol. La proximidad. ¿Un nuevo valor urbano? Ciudades, v. 17, n. 1, p. 99-120, 2014. https://doi.org/10.24197/ ciudades.17.2014 
31. NATERA, Juan; GÓMEZ, Néstor. Diferenciación socio residencial en el aglomerado del Gran Santa Fe (Argentina) a comienzos del siglo XXI. Revista Universitaria de Geografía, v. 16, n. 1, p. 99-124, 2007.

32. ORTÍZ, Jorge; SCHIAPPACASSE, Paulina. Dimensiones latentes de la diferenciación del espacio social en una metrópolis latinoamericana. El caso del Gran Santiago. Geographicalia, n. 36, p. 111-130, 1998.

33. PRÉVOT-SCHAPIRA, Marie. Fragmentación espacial y social: conceptos y realidades. Perfiles Latinoamericanos, n. 19, p. 33-56, 2001.

34. RODRÍGUEZ, Jorge. Segregación residencial socioeconómica iqué es? ¿Cómo se mide? ¿Qué está pasando? ¿̇lmporta? Serie Población y Desarrollo 16, Santiago de Chile: CEPAL, 2001.

35. RODRÍGUEZ, Jorge; ARRIAGADA, Camilo. Segregación residencial en la ciudad latinoamericana. Revista EURE, v. 30, n. 89, p. 5-24, 2004. http://dx.doi. org/10.4067/S0250-71612004008900001

36. SABATINI, Francisco; BRAIN, Isabel. La segregación, los guetos y la integración social urbana: mitos y claves. Revista EURE, v. 34, n. 103, p. 5-26, 2008. http:// dx.doi.org/10.4067/S0250-71612008000300001

37. SABATINI, Francisco; CÁCERES, Gonzalo; CERDA, Jorge. La segregación residencial en las principales ciudades chilenas. Revista EURE, v. 27, n. 82, p. 21 42, 2001. http://dx.doi.org/10.4067/S0250-71612001008200002

38. VALDEBENITO, Carlos. Segregación y fragmentación urbana de tipo socioeconómica: el caso de Viña del Mar en el área metropolitana de ValparaísoChile. In: SOTO, Marcela; ÁLVAREZ, Luis. Fenómenos informales clásicos en la megaciudad latinoamericana. Valparaíso: UTFSM Editorial, 2011. p. 104-123.

39. VALDEBENITO, Carlos. La huella socioeconómica y demográfica en la estructura residencial de las ciudades medias de Latinoamérica: el caso de Viña del Mar-Chile en la década 1992-2002. Scripta Nova - Revista Electrónica de Geografía y Ciencias Sociales, v. 43, n. 492, 2014.

40. VALDEBENITO, Carlos. De actores a agentes en la ciudad neoliberal periférica. La huella de las cooperativas de vivienda cerradas en la diferenciación socioresidencial de la ciudad de Viña del Mar, Chile. In: XV Coloquio Internacional Geocrítica, 7 a 12 de mayo de 2018, Barcelona. Anales... Barcelona: Universitat de Barcelona, 2018.

41. VILAGRASA, Joan. Los debates sobre Pobreza Urbana y Segregación Social en Estados Unidos. Scripta Nova - Revista Electrónica de Geografía y Ciencias Sociales, v. 4, n. 76, 2000. 
